Original Research Article

\title{
A comparative study between retrospective and prospective assessment of rationality and effectiveness of snake bite management in a tertiary care teaching hospital
}

\author{
Sonai Mandal ${ }^{1}$, Ananya Mandal ${ }^{2}$, Tamoghna Maity ${ }^{1}$, Somenath Das $^{1}$
}

\begin{abstract}
${ }^{1}$ Department of Pharmacology, Bankura Sammilani Medical College, Bankura, West Bengal, India

${ }^{2}$ Department of Pharmacology, Nilratan Sircar Medical College, Kolkata, West Bengal, India
\end{abstract}

Received: 22 May 2019 Accepted: 29 May 2019

*Correspondence to: Dr. Ananya Mandal, Email: drananyamandal@ gmail.com

Copyright: (C) the author(s), publisher and licensee Medip Academy. This is an openaccess article distributed under the terms of the Creative Commons Attribution NonCommercial License, which permits unrestricted noncommercial use, distribution, and reproduction in any medium, provided the original work is properly cited.

\begin{abstract}
Background: Snake bite envenomation remains one of the commonest causes of mortality and morbidity in rural West Bengal. Objective of present study was to compare the rationality, effectiveness and adherence to standard treatment guidelines of Government of West Bengal of snake bite management over the high-risk periods of two years.

Methods: This was a comparative study of snake bite management between 2016 and 2018 (over April to September) during peak risk season in a tertiary care teaching hospital in rural Bengal. The data was collected retrospectively from the treatment records in 2016 and from patients admitted with history of snake bite in the Medicine ward, ICU and ITU in 2018.

Results: Records from 2016 yielded 177 snake bite patients (144 venomous and $82.5 \%$ neurotoxic envenomation) and in 2018, 190 cases (114 venomous and $71.1 \%$ neurotoxic). There was delay in presentation ( $>2$ hours) in almost $80 \%$ cases in both years and $2.56 \%$ had anaphylactic reaction following ASV administration in 2018 against $3.15 \%$ in 2016 . Ventilator support was necessary for $12 \%$ patients in 2018 . Overall percentage of mortality reduced from $6.70 \%$ to $3.2 \%$ in 2018 . Adherence to snake bite management STG was seen in $81.8 \%$ of the retrospective records vis a vis $80 \%$ cases in prospective analysis $(\mathrm{p}=0.21)$. Conclusions: The survival rate in venomous snake bite is found to be high in this institution over last two seasons of snake bite. The practice of snake bite management is found to be adherent with standard protocol in most cases.
\end{abstract}

Keywords: Anti snake venom, Hematotoxic envenomation, Neurotoxic envenomation, Snake bite, Standard treatment guidelines

\section{INTRODUCTION}

Envenomation and deaths due to snakebite has always remained a life-threatening public health problem in rural tropical countries like India. The global estimate of snakebite envenomation is 421,000 to $1,842,000$ cases with 20,000 to 94,000 death each year, though the actual incidence varied a lot and may even be higher due to underreporting. India contributes a portion of the global total with 45,900 annual snakebite deaths nationally $(99 \%$
CI 40,900 to 50,900 ) which also is expected to be underestimated due to an inclination of village-based traditional therapists..$^{1,2}$

Snake bite is a condition that disproportionately affects the poor community amounting to a huge treatment cost. Further the pharmaceutical companies show a minimal interest in it due to less sustainable profit. There is also meagre health agencies and research funding which has led to ASV scarcity. In June 2019, the World Health 
Organization (WHO) enlisted snakebite envenomation as a category A neglected disease aiming to boost the snakebite management globally especially in developing countries. $^{3}$ In India, West Bengal also remains as one of the most prevalent and high mortality state due to snakebite, apart from Uttar Pradesh (UP), Tamil Nadu, Bihar, and Maharashtra. ${ }^{2}$

A community-based survey in West Bengal had shown a high annual mortality rate of 16.4 deaths/100,000 but extrapolating such data for a national data cannot be justified due to the wide variation in the geographical distribution of snakes and a poor reporting system. ${ }^{2}$ People living in rural areas are mostly involved in agriculture which poses snakebite to be an occupational hazard, often affecting adult males in the professionally active age range. Therefore snakebite has a considerable economic impact especially in an economically vulnerable rural population. ${ }^{1,2}$

Till date, snake antivenom or antisnake venom (ASV) remains the only specific treatment for snake envenomation. Polyvalent ASV is produced in India by hyper immunising horses against venoms of four common species of snake believed to be responsible for most deaths due to snakebite envenomation. However, this concept is recently being challenged due to discovery of other important venomous species according to geographical distribution. To address the issue of low access to ASV, it was included by WHO in the Model List for Essential Medicines, encouraging and ensuring adequate national ASV stocks to be maintained by all countries. ${ }^{3-5}$

Lack of first aid, medical care and public awareness added with scarcity of ASV and strong belief in traditional cultural attitudes towards snakes and snake bites has worsened the picture in India. Moreover, injudicious use of ASV will not only risk the safety of the patient to adverse drug reactions but will also magnify the scarcity of ASV. ${ }^{6}$

As a result of the non-uniform ASV doses and low compliance to the existing treatment protocols the Ministry of Health and Family Welfare (Government of India) felt the need for formulation of the National Protocol on Snake Bite Management besides the WHO Guidelines by in June 2016. That focused not only an effective management of snakebites but also on training programs for care providers.

This was formulated as the Standard treatment guidelines, Management of Snake Bite - Quick Reference Guide January 2016. Management of Snake Bite. Quick Reference Guide January 2016. Ministry of Health and Family Welfare. Government of India). ${ }^{7}$ The present study was carried out to compare rationality, effectiveness and adherence to STG 2016 between 2016 considered as the retrospective year before implementation of STG and 2018 as the prospective year in a tertiary care hospital of Bankura, West Bengal.

\section{METHODS}

This was a hospital based comparative, observational and prospective study. The study was conducted in the month of April to September in year of 2016 and 2018. The following months were chosen as there is more incidence of snakebite in pre-monsoon and monsoon season relative to the winter season when they go for hibernation.

The study was carried out in the department of Medicine and Pharmacology of Bankura Sammilani Medical College which caters mostly rural and semiurban population from three districts including two adjacent districts of Purulia and Paschim Medinipur. Patients who were admitted in Medicine ward, ICU and ITU with a history of snakebite (history or suggestive bite mark) and are willing to give consent for participation in the study were included in the study. All snakebite patients admitted fulfilling the above criteria within the study period. Patients who were brought dead to the hospital were not included in the study.

After attaining permission from Institutional Ethics Committee, data collection for retrospective year 2016 was initiated from institution's central record section after obtaining due permission from the concerned authority. The bed head ticket of all patients admitted in medicine ward, ITU and ICU of this hospital with a history of snakebite within the study period were included in the study. All relevant data regarding clinical manifestation, laboratory investigations and management along with the outcome were recorded in the case record form.

The data for prospective year 2018 was done by interviewing and by follow up visits till discharge or death of the concerned patient. Clinical features along with and laboratory findings, were also taken into account. Data collection was started after attaining permission from Institutional Ethics Committee and obtaining duly signed informed consent document from the patient or their relative. Elaborate history of demographic details, snake type, snakebite time, site of bite, bite to ASV time, clinical findings were taken. The management given by the attending physician and the laboratory test details were recorded. All the enrolled patient including those needing dialysis and ventilator support were followed up daily till their discharge or death.

All the data were compiled in a Microsoft Excel sheet and were analysed for descriptive statistics with statistical software SPSS version 22. All the treatments including both retrospective and prospective snakebite cases were analysed whether they adhered to the standard treatment guidelines national STG 2016. The rationality of each treatment were analysed individually and effectiveness of National STG on snakebite in prospective year was evaluated. Rationality of the ASV treatment and adherence to protocol between the two groups were compared using Chi square test with a $\mathrm{p}$ value of less than 0.05 taken to be significant. 


\section{RESULTS}

In retrospective year a total of 177 bedhead tickets of patients admitted in the hospital within the study period with a history of snakebite were included in the study. Among them 145 cases $(81.80 \%)$ were found to venomous and the rest $32(18.20 \%)$ were treated as nonvenomous.

In prospective year 2018, a total 190 patients were admitted in Medicine ward, ITU, ICU with a history of snakebite within the time frame. $60.30 \%$ of the patients were treated as venomous bite while the rest 76 patients $(39.70 \%)$ were treated as nonvenomous bite, for which they were under close observation and managed symptomatically. The bite types in each of the prospective and restrospective studies are depicted in Figure 1.

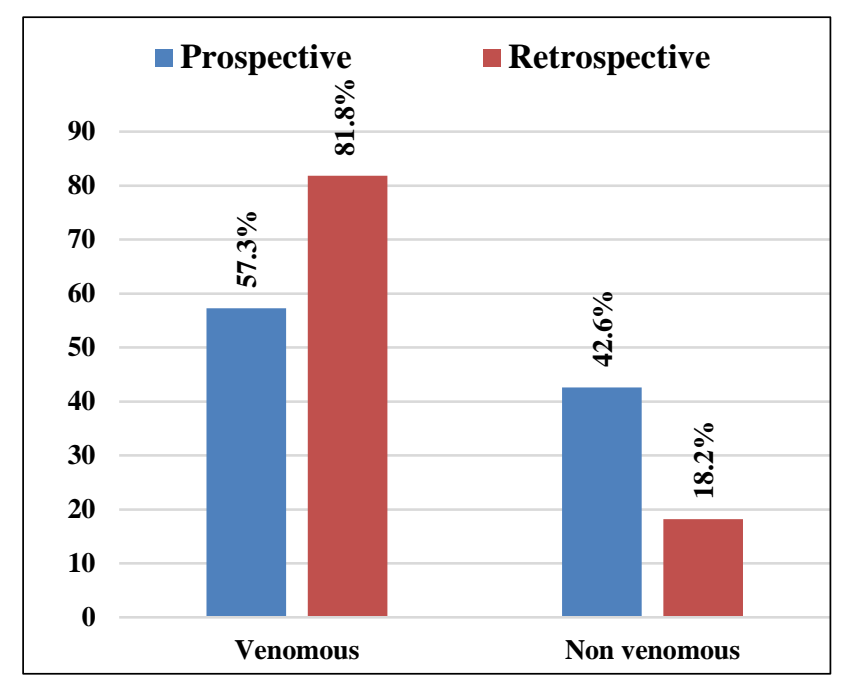

Figure 1: Percentage of venomous and non-venomous bites in prospective and retrospective studies.

In the retrospective study, among the venomous bites, we found $82.50 \%$ were neurotoxic followed by $17.50 \%$ haematotoxic and no case was registered showing features of both neuro and hematotoxicity. On the other hand in prospective year we had $71.1 \%$ as neurotoxic, $27.90 \%$ hematotoxic and $1 \%$ as neuro-hematotoxic case.

Maximum percentage of snakebite victims in both retrospective as well as prospective year were males, accounting for $66.66 \%$ and $72.1 \%$ respectively. In both the years authors found the adult age group to be more prone. Within 18-60 years age group the incident was $79.3 \%$ versus $78 \%$ in retrospective and prospective years. However the incidence was found less in less than 18 years age group with $15.3 \%$ versus $14.8 \%$ respectively. It was found lesser in above 60 years of age group. (5.4\% vs7.2\%)

Almost $80 \%$ percent of the snakebite victim encountered within the study time frame belonged to rural area in both the years. (rural- $79.3 \%$ vs $76.3 \%$, urban $20.7 \%$ vs $23.7 \%$ ). In present study the farmers and the fieldworkers were affected more with an incidence of $35.3 \%$ and $31.3 \%$ in retrospective and prospective years respectively followed by household workers, students and other occupation.

The incidence of snakebite patients referred from primary health centre increased from $23 \%$ in retrospective year to $38 \%$ in prospective year. The rest came to the hospital directly. The time required to reach the nearest health centre was greater than 2 hours in majority of cases in both the years. Though the percentage decreased in prospective year from $84.1 \%$ to $70 \% .13 .9 \%$ snakebite victims reached the hospital within 1-2 hours in retrospective year against $24.6 \%$ in prospective year. Very few percentage less than $5 \%$ reaches hospital within 1 hour in both the years.

The most common site of bite was foot followed by legs, upper limbs and other body parts in both the years. Almost $40 \%$ of the patients admitted in hospital presented with bite on foot. In both the years the prevalence of snakebite happened to be occurring during early morning or during sleeping. It almost accounted for $49 \%$ in retrospective year against $56 \%$ in prospective year.

In retrospective year majority of the snakebite victims $(84.1 \%)$ took more than 2 hours to reach the nearest hospital. Unfortunately, the percentage didn't vary much as $70.1 \%$ still took more than 2 hour time to reach the hospital. Very low percentage of patients i.e. less than $5 \%$ reached hospital in less than an hour. Though $24.6 \%$ snakebite victims were brought to hospital within 2 hours in prospective years but only $13.9 \%$ turned up in retrospective year.

Local swelling (38\%) was the most common presenting feature followed by ptosis and dysphagia (38\%) in retrospective year. While nearly $40 \%$ presented with local swelling followed by ptosis and dysphagia. $19.3 \%$ had a respiratory distress in retrospective year against $24.5 \%$ in prospective year. Among them 18\% were shifted to ventilator support in prospective year. A good proportion of patients complained of more than one feature at the time of admission.

In retrospective year all 145 patients with venomous snakebite and 13 patients among nonvenomous received ASV. Whereas in prospective year all 109 venomous snakebite and 11 nonvenomous snakebite received ASV showing a reduction in use of ASV in nonvenomous snakebite cases. The adherence to ASV indication in retrospective versus prospective year was $71.4 \%$ to $81.3 \%$ respectively. There was a decreasing trend of usage of ASV in primary health care centre in snakebite cases when compared with two years. It decreased from $93 \%$ of ASV usage in retrospective year to $78 \%$ in prospective year. The average dose of ASV received outside was variable in both years with an average of 6.9 vials in retrospective year and 8.9 vials in prospective year. The average utilisation of ASV decreased from an average of $30.78 \pm 8.6$ vials in retrospective study to $21.54 \pm 3.71$ vials in prospective study. 
Table 1 depicts the average ASV vial requirement in different types of snakebite according to the year.

Adherence to ASV dosage in accordance to the guideline was found to be $68.3 \%$ in retrospective year whereas $76.8 \%$ was found in prospective year. Further $71.4 \%$ adherence was found regarding ASV indication in retrospective year and $81.3 \%$ was in prospective year. On comparing the two years using Chi square test, rationality of ASV use and adherence to STG was found to be statistically similar in both years ( $p=0.18$ and $p=0.21$ respectively). The results are depicted in Figure 2.

Table 1: Average ASV vial requirement in different types of snakebite according to the type of study.

\begin{tabular}{|lll|}
\hline $\begin{array}{l}\text { Types of } \\
\text { snakebite }\end{array}$ & $\begin{array}{l}\text { Retrospective } \\
\text { study } \\
(\text { Average } \pm \text { SD) }\end{array}$ & $\begin{array}{l}\text { Prospective } \\
\text { study } \\
\text { (Average } \pm \text { SD) }\end{array}$ \\
\hline Neurotoxic & $24.9 \pm 11.54$ & $18.19 \pm 7.3$ \\
\hline Vasculotoxic & $28.2 \pm 9.9$ & $20.54 \pm 9.1$ \\
\hline Mixed & $23.1 \pm 6.5$ & $18.7 \pm 5.33$ \\
\hline
\end{tabular}

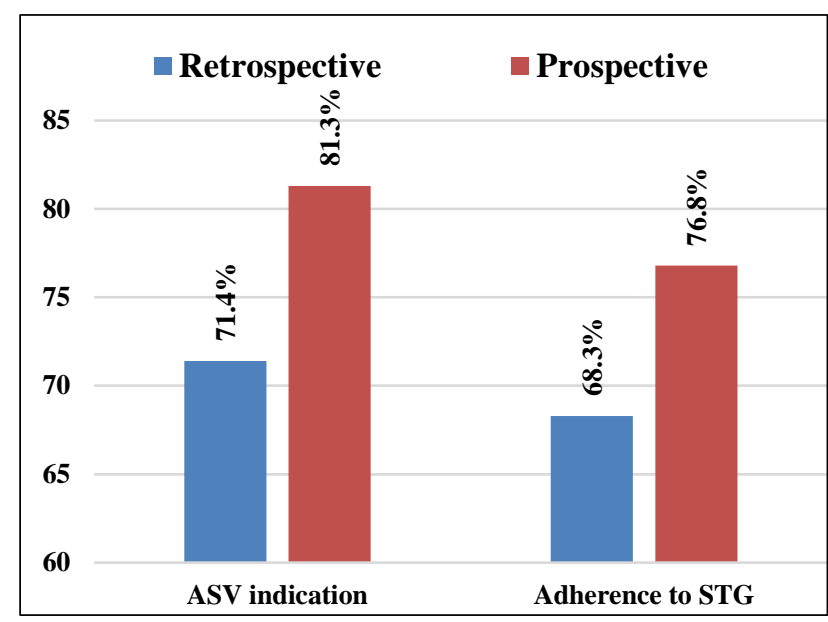

Figure 2: Adherence to ASV indication and Standard treatment guidelines in retrospective and prospective studies.

To counter the anaphylactic reactions due to ASV usage, dexamethasone and hydrocortisone injections were used in retrospective year. Among them 60\% received dexamethasone, $22 \%$ received hydrocortisone and rest received both. Whereas in prospective year all 121 patients received ASV with hydrocortisone injection as prophylaxis. While 33\% among them also received adrenaline along with injection hydrocortisone.

Authors noted that $20.7 \%$ snakebite victims received atropine and neostigmine in retrospective year whereas $86 \%$ received it in prospective year in accordance to the mentioned indication. Injectable antibiotics like amoxicillin-clavulanic acid, ampicillin, ceftriaxone and metronidazole were used in $70.3 \%$ of patients of snakebite in retrospective year. However it decreased in prospective year to $65 \%$. Almost all patient either presenting directly to
BSMC or referred from primary health centre received Injection Tetanus toxoid in both the years.

According to the guidelines, 20 Whole Blood Clotting Time (20 WBCT) being considered the most reliable bedside test requiring no speciality. If $20 \mathrm{WBCT}$ remains negative, then the patient's blood is retested every hour for first three hours and then 6 hourly for 24 hours till test results are positive or other clinical evidence to ascertain ASV dose is found. If $20 \mathrm{WBCT}$ is positive than it should be repeated after 6 hours of administration of ASV which is same in case of neurotoxic snakebite. We found $89 \%$ and $94.66 \%$ adherence in retrospective and prospective years respectively.

151 patients were discharged on complete recovery in respect to 174 patients in prospective year. Among them majority of the snakebite victims were discharged within 3 to 4 days in both the years. Figure 3 shows that average number of hospital stay in days in both the study groups.

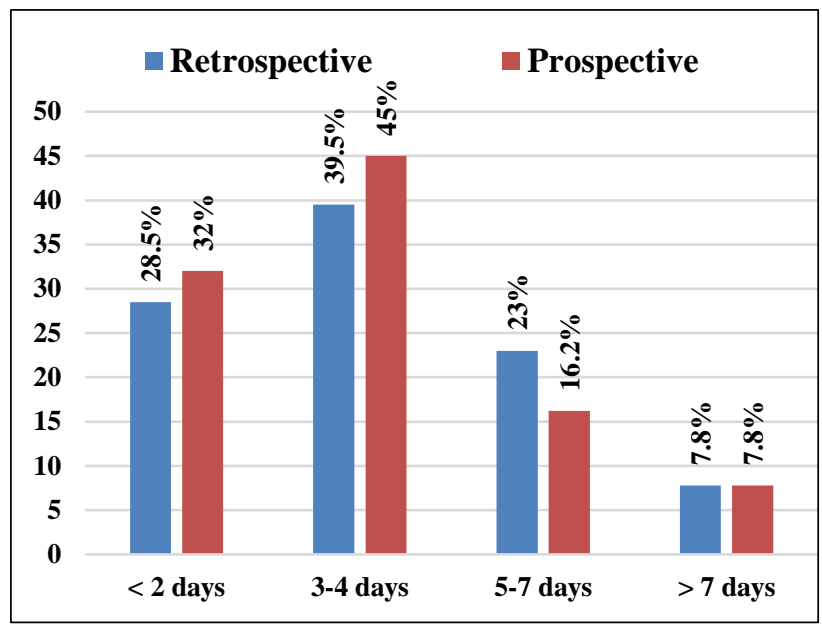

Figure 3: Average number of hospital stays in both groups of patients.

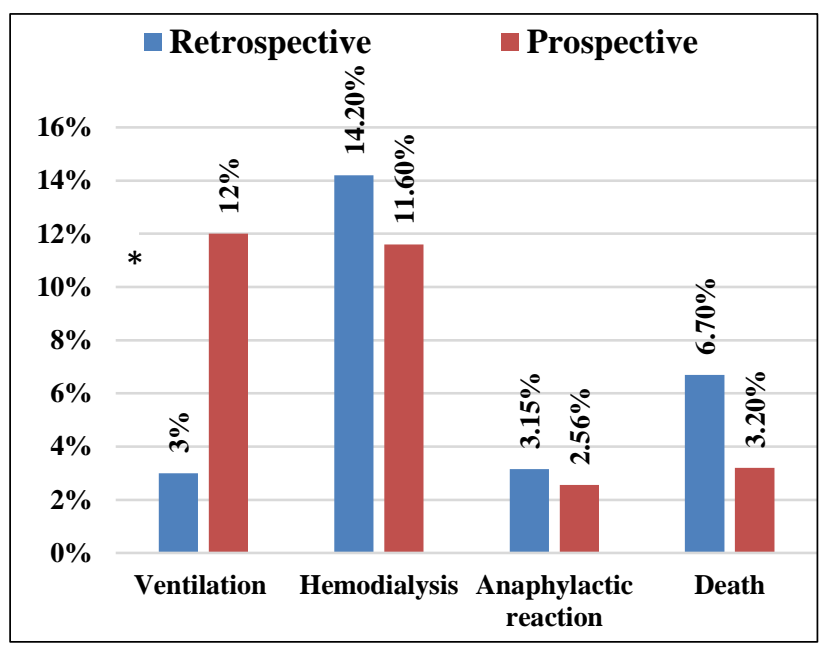

*Statistically significant

Figure 4: Patient outcome after snake bite management in both study groups. 
Authors encountered 5 deaths due to respiratory failure and 1 death due to acute kidney injury (AKI) in prospective year. The incident of death in retrospective year was 12 , among which 9 patients succumbed due to respiratory failure and the rest 3 due to AKI. Figure 4 shows the patient outcome from both the retrospective and prospective studies.

\section{DISCUSSION}

Snakebite remains one of the most neglected health problems in India whose true burden is also systematically underestimated. Due to disparity in treatment modalities, limitations of resources, delay in arriving to the nearest medical facility along with a wide variation in treatment approach has made the scenario unfavourable. In retrospective year we found a lesser number of snakebite envenomation patients than prospective year highlighting the fact that the actual number of snakebite victims was underestimated.

It was unfortunate to find that the working age group of 18 to 60 years was the most affected group in both the years. They gave a history of snakebite while working in field as agriculture is the rural livelihood of most people of Bankura district. In present study authors found the young agricultural male being the most affected population thus affecting the economically vulnerable patients as seen by other researchers also. ${ }^{1,2}$

The percentage of venomous and nonvenomous snakebite patients in retrospective year was $80 \%$ and $20 \%$ respectively which was very similar to findings of other studies undertaken in this institution and other institution of west Bengal. ${ }^{8,9}$ The percentage for venomous snakebite slightly decreased in prospective year to almost $60 \%$ like another study conducted in same institution due to the implementation of national STG the treatment initiation with ASV greatly depended on a judicious screening of snakebite patients considering clinical features and observation rather than infusing ASV to everyone. ${ }^{10,11}$ Introduction of National STG was aimed in attenuating the scarcity of ASV by its judicious application which was fulfilled by giving training to the physicians in this institution. With such approach it was evident that the imprudent use of ASV was minimised.

Among the venomous bites, we found an extensive number of cases for neurotoxic envenomation in both the years as West Bengal happens to be the natural territory of common krait, one of the venomous species. Due to its nocturnal habit and finding refuge in human house hold during rainy season the bite generally occurs at night with little or no pain complaint by the victim. ${ }^{12}$ This leads to more fatalities due to delayed onset of symptoms and delayed commencement of treatment. Though the ratio of neurotoxic to hematotoxic envenomation varied between two groups but neurotoxic had a larger proportion depicting a similar likeness to other studies. ${ }^{4,8,11}$
There was a gross deficit in awareness regarding early presentation of snakebite victim the outcome of which was a delayed presentation of snakebite victim to nearest health facility. Quack and indigenous treatments often take up a good number of hours before a patient presents to the health care facility. Almost $85 \%$ took more than 2 hours to reach the nearest hospital in retrospective year. The percentage decreased in prospective year to $70 \%$ reinforcing the fact that more initiatives are needed in increasing the awareness regarding snakebite in rural population. ${ }^{4,6,11,13}$ Snakebites in the early morning and foot being the commonest site of bite followed by legs and upper limbs was seen in both years as has been recorded in other studies. ${ }^{4,11}$

Polyvalent ASV remained the mainstay of snakebite victims in our country knowing the fact that it is only able to neutralise the free unbound toxins. It remains ineffective to the bound portion, so timely institution of ASV is recommended as there are studies proving to have direct correlation with patients outcome.7,14,15 One of the objectives of implementation of National and WHO STG guideline on snakebite was to practice a rational use of ASV combat it scarcity. ${ }^{7,13}$ The practice of ASV usage in primary health centre to every patient presenting with a history of snakebite decreased in prospective year when compared with the retrospective year. The unrestrained use of ASV in retrospective year unearthed the fact mentioned in various other studies that owing to fear of death of the patients, the attending physician resolve to use ASV injudiciously. ${ }^{13}$

A systemic review of randomized trials comparing high and low dose of ASV concluded that low dose have the similar efficacy and cost effective in treatment of snakebite poisoning but National STG proposes to start with 10 vials as infusion over 30 minutes for neurotoxic envenomation followed by a repeat 10 vial dose of ASV if no improvement is seen. ${ }^{7,11,14}$ Low dose infusion therapy and high dose intermittent bolus therapy, both are followed in case of vasculotoxic snakebite. Repeat dose by 6 vials 6 hourly as bolus therapy is given till clotting time normalizes. The average use of ASV in both neurotoxic as well as hematotoxic reduced in prospective year when compared with retrospective year thus proving that a proper implementation of national STG on snakebite can bring down the needless ASV consumption. We found a fairly high level of adherence to National STG in respect to ASV use in accordance to the mentioned indication and also with the ASV dosage in prospective year.

As mentioned earlier 20 WBCT should be monitored hourly for first 3 hours and then every 4 hours for remaining 24 hours. If the test results shows non clotting, then it is to be repeated 6 hours after administration of ASV.7In retrospective year we found an appreciable adherence in regards to monitoring by 20 WBCT which reached to $95 \%$ in prospective year. 
Anticholinesterase (e.g. neostigmine) are administered with the aim of reversing the neuroparalysis common in krait bite and thus expected to have a significant role in patient outcome. There are studies having an inconsistent result of it efficacy in avoiding the need for ventilation but National STG and WHO recommended it in neuroparalytic type of snakebite. ${ }^{7,15}$ Adherence to atropine and neostigmine administration in accordance to the indication was $20.7 \%$ in retrospective year whereas a remarkable increase of $86 \%$ was found in prospective year. Earlier the physicians were unacquainted with the benefits of atropine neostigmine injection in snakebite management accounting for low adherence in previous years which corroborated with the findings of other studies. ${ }^{8}$ Corticosteroids does not have a direct role in combating snake venom but its administration prevents from anaphylaxis reaction likely to occur from administration of snake venom. Dexamethasone has an antagonizing activity against myotoxicity and inflammation caused by snake venom. ${ }^{16}$ In the retrospective year majority received dexamethasone followed by hydrocortisone while all patients in prospective year received hydrocortisone.

Mortality reduced in prospective year when compared to retrospective year. Even the frequency of anaphylaxis was also reduced in prospective year proving that implementation of National STG on snakebite reduces both mortality and ASV consumption corroborating with other studies. ${ }^{11}$

\section{CONCLUSION}

Authors can conclude from out study that a high level of adherence to the Standard Treatment Guidelines was seen in authors set up leading to a significantly reduced mortality due to snake bites despite the high incidence of venoumous bites. In the prospective study there was a significantly high adherence to WBCT monitoring, supportive management such as use of atropine and neostigmine as well as ventilation. Present study paves the way to better snake bite management at our rural set up and we aim at mass awareness to reduce delay in hospital visit after the bite especially due to quack and indigenous treatment.

\section{Funding: No funding sources}

Conflict of interest: None declared

Ethical approval: The study was approved by the Institutional Ethics Committee

\section{REFERENCES}

1. Avau B, Borra V, Vandekerckhove P, De Buck E. The treatment of snake bites in a first aid setting: a systematic review. PLoS Negl Trop Dis 2016;10(10): e0005079.

2. Mohapatra B, Warrel DA, Suraweera W, Bhatia P, Dhingra N, Jotkar RM et al. Million Death Study Collaborators. Snakebite mortality in India: a nationally representative mortality survey. PLoS Negl Trop Dis. 2011;5(4):e1018.

3. Chippaux JP. Snakebite envenomation turns again into a neglected tropical disease! Journal of Venomous Animals and Toxins including Tropical Diseases 2017;23(1):38.

4. Sarkhel S, Ghosh R, Mana K, Gantait Ket. A hospital based epidemiological study of snakebite in Paschim Medinipur district, West Bengal, India Toxicol Rep. 2017;4:415-9.

5. WHO Model Lists of Essential Medicines. Available at:

https://www.who.int/medicines/publications/essential medicines/en/ Accessed on 28th March 2019.

6. Ghosh S, Maisnam I, Murmu BK, Mitra PK, Roy A, Simpson ID. A locally developed snakebite management protocol significantly reduces overall anti snake venom utilization in West Bengal, India. Wilderness Environ Med. 2008;19(4):267-74.

7. Standard Treatment Guidelines on Snakebite. Directorate General of Health Services, Ministry of Health and Family Welfare, Government of India With Technical Support from National health Systems Resource Centre; 2016. Available at: http://www.nhm.gov.in/nrhm-instate/520-standardtreatment-guidelines.html Accessed on 28th March 2019.

8. Dan S, Mandal A, Das A, Chakrabarty S, Gangopadhyay T. A 6 months retrospective observational study to assess the rationality and effectiveness of snake bite management in a tertiary care teaching hospital of rural Bengal, India. Int $\mathbf{J}$ Basic Clin Pharmacol. 2018;7:2047-52.

9. Pal A, Mukherjee S. Clinical profile and outcome of snake bite in patients attending a tertiary care hospital in Bankura, West Bengal IOSR Journal of Dental and Medical Sciences (IOSR-JDMS). 2018;17:34-9.

10. Majumder D, Sinha A, Bhattacharya SK, Ram R, Dasgupta U, Ram A. Epidemiological profile of snake bite in South 24 Parganas district of West Bengal with focus on underreporting of snake bite deaths. Indian J Public Health. 2014;58(1):17-21.

11. Mandal S, Mandal A, Chatterjee Y, Das S, Kundu S, Naser SM. A prospective analysis of adherence to standard treatment guidelines in management of snakebite in a tertiary care teaching hospital of rural Bengal, India. Int J Basic Clin Pharmacol. 2019;8:84955.

12. Kulkarni ML, Anees S. Snake venom poisoning: experience with 633 cases. Ind Pediatrics. 1994;31:1239-43.

13. Daswani BR, Chandanwale AS, Kadam DB, Ghonghane DB, Ghorpade VS, Manu HC. Comparison of Different Dosing Protocols of AntiSnake Venom (ASV) in Snake Bite Cases. Journal of Clinical and Diagnostic Research. 2017;11(9):FC17FC21.

14. Rashmi RD, Jhuma S, Nishanth D. High-dose versus low-dose antivenom in the treatment of poisonous 
snake bites: A systematic review. Indian J Crit Care Med. 2015;19(6):340-9.

15. Anil A, Singh S, Bhalla A, Sharma N, Agarwal R, Simpson ID. Role of neostigmine and polyvalent antivenom in Indian common krait (Bungarus caeruleus) bite. Journal of Infection and Public Health. 2010;3(2):283-7.

16. Patrão-Neto FC, Tomaz MA, Strauch MA, MonteiroMachado M, Rocha-Junior JR, Borges PA, et al. Dexamethasone antagonizes the in vivo myotoxic and inflammatory effects of Bothrops venoms. Toxicon. 2013 Jul 1;69:55-64.

Cite this article as: Mandal S, Mandal A, Maity T, Das S. A comparative study between retrospective and prospective assessment of rationality and effectiveness of snake bite management in a tertiary care teaching hospital. Int J Basic Clin Pharmacol 2019;8:1547-53. 